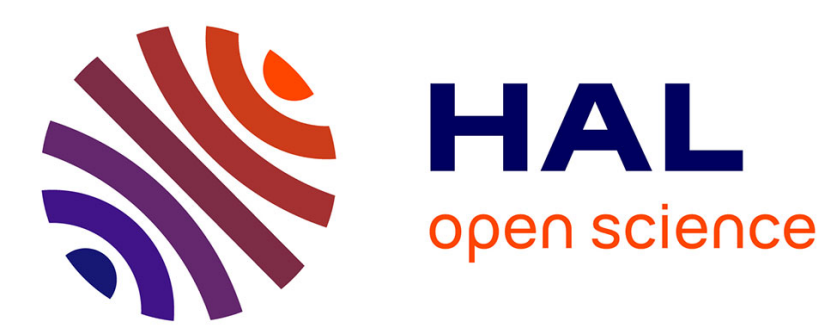

\title{
Powerful Parallel and Symmetric 3D Thinning Schemes Based on Critical Kernels
}

\author{
Gilles Bertrand, Michel Couprie
}

\section{To cite this version:}

Gilles Bertrand, Michel Couprie. Powerful Parallel and Symmetric 3D Thinning Schemes Based on Critical Kernels. Journal of Mathematical Imaging and Vision, 2014, 48 (1), pp.134-148. 10.1007/s10851-012-0402-7 . hal-00828450

\section{HAL Id: hal-00828450 \\ https://hal.science/hal-00828450}

Submitted on 31 May 2013

HAL is a multi-disciplinary open access archive for the deposit and dissemination of scientific research documents, whether they are published or not. The documents may come from teaching and research institutions in France or abroad, or from public or private research centers.
L'archive ouverte pluridisciplinaire HAL, est destinée au dépôt et à la diffusion de documents scientifiques de niveau recherche, publiés ou non, émanant des établissements d'enseignement et de recherche français ou étrangers, des laboratoires publics ou privés. 


\title{
Powerful Parallel and Symmetric 3D Thinning Schemes Based on Critical Kernels *
}

\author{
Gilles Bertrand and Michel Couprie \\ Université Paris-Est, Laboratoire d'Informatique Gaspard-Monge, ESIEE Paris \\ Cité Descartes, BP 99, 93162 Noisy-le-Grand Cedex France \\ g.bertrand@esiee.fr,m.couprie@esiee.fr
}

September 12, 2012

\begin{abstract}
The main contribution of the present article consists of new 3D parallel and symmetric thinning schemes which have the following qualities:

- They are effective and sound, in the sense that they are guaranteed to preserve topology. This guarantee is obtained thanks to a theorem on critical kernels;

- They are powerful, in the sense that they remove more points, in one iteration, than any other symmetric parallel thinning scheme;

- They are versatile, as conditions for the preservation of geometrical features (e.g., curve extremities or surface borders) are independent of those accounting for topology preservation;

- They are efficient: we provide in this article a small set of masks, acting in the grid $\mathbb{Z}^{3}$, that is sufficient, in addition to the classical simple point test, to straightforwardly implement them.
\end{abstract}

Keywords: Thinning algorithm, skeleton, parallel algorithm, critical kernel, cubical complex, simple point, collapse.

\section{Introduction}

Computing the skeleton of a 3D shape is a fundamental step in several applications dealing with shape analysis, shape recognition, registration, visualization, animation, etc. A fundamental property of skeletons is topology preservation: a skeleton must have the same topological characteristics as the original shape.

*This work has been partially supported by the "ANR-2010BLAN-0205 KIDICO” project.
In discrete grids $\left(\mathbb{Z}^{2}, \mathbb{Z}^{3}, \mathbb{Z}^{4}\right)$, a topology-preserving transformation can be defined thanks to the notion of simple point [25]: intuitively, a point of an object (a subset of $\mathbb{Z}^{d}$ ) is called simple if it can be deleted from this object without altering topology. Let us illustrate this notion by Fig. 1, which displays a same subset of $\mathbb{Z}^{2}$ under two usual representations: as a set of points (left), and as a set of pixels (right). In this example, the pixels (or points) $a, b, c$ are simple but $x, y, z, t$ are not. This notion, pioneered by Duda, Hart, Munson [18], Golay [20] and Rosenfeld [43], has since been the subject of an abundant literature. In particular, local characterizations of simple points have been proposed (see $\mathrm{e} . \mathrm{g}$. $[13,17])$, on which efficient implementation of thinning procedures are based.
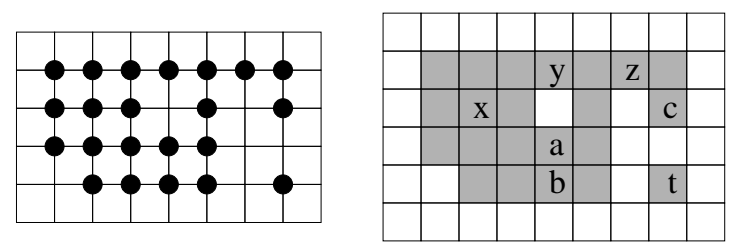

Figure 1: Illustration of 2D simple points/pixels. The set $X$ is made of the points represented as black discs on the left, and by gray pixels on the right. The points/pixels $a, b, c$ are simple while $x, y, z, t$ are not: deleting $x$ would create a hole in $X$, deleting $y$ would suppress a hole, deleting $z$ would split a connected component, and deleting $t$ would suppress a connected component.

The most "natural" way to thin an object consists of removing some of its border points in parallel. By parallel, we mean that the same operation is executed simul- 


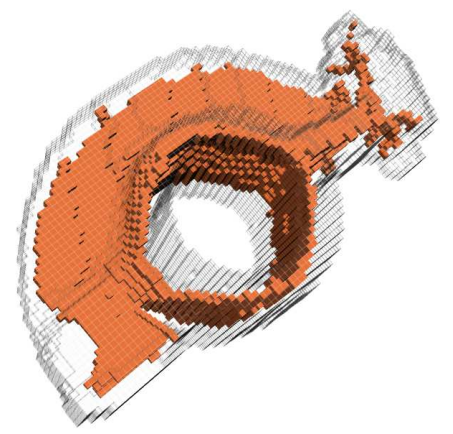

(a)

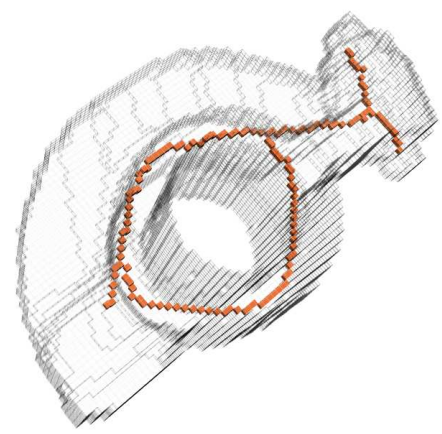

(b)

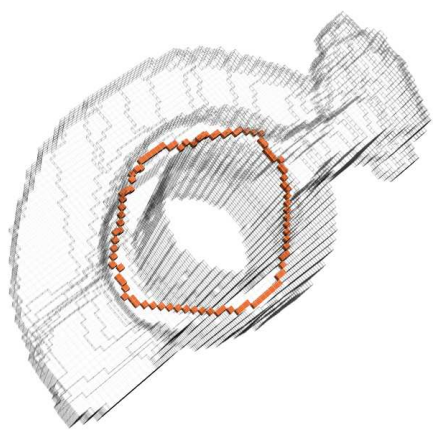

(c)

Figure 2: Different kinds of skeletons: (a) surface skeleton, (b) curvilinear skeleton, (c) minimal skeleton.

taneously and independently for each image point. By repeating such a procedure until stability, one can obtain a well-centered "skeleton" of the original object (see Fig. 2). Furthermore, parallel thinning algorithms tend to produce skeletons which are more robust to small variations of shape contours, in comparison with sequential algorithms which must make arbitrary choices regarding the order of the processing of points.

However, parallel deletion of simple points does not, in general, guarantee topology preservation: see for example Fig. 1 where the points $a$ and $b$ are both simple, and removing these two points simultaneously would merge two background components. In fact, such a guarantee is not obvious to obtain, even for the 2D case (see [16], where fifteen published parallel thinning algorithms are analyzed, and counter-examples are shown for five of them).

For the 2D case, A. Rosenfeld introduced in [44] a method that consists of dividing each thinning step into four substeps. Each of these substeps considers as candidate for deletion, only the simple points that have no neighbor belonging to the object in one of the four main directions (north, south, east, west) and have at least two 8-neighbors belonging to the object. However, this so-called directional strategy cannot be straightforwardly extended to 3D. In this case, the six main directions are north, south, east, west, up and down. In Fig. 3, the voxels $x, y$ are simple voxels that have no neighbor belonging to the object in the direction "up", but if we remove them in parallel, the object splits.

Some authors (see e.g. [9, 33, 39, 38]) have proposed thinning algorithms based on the so-called subfield strategy, a general strategy which permits the parallel deletion of certain simple points. It consists of considering, in each substep, only simple points that belong to a given subgrid (also called subfield). For example in 2D (resp. 3D), four (resp. eight) disjoint subfields may be defined by saying that two points belong to the same subfield if the parity of each of their coordinates is the same. Variants with four or even two subfields, in 3D, have also been proposed; but additional conditions must be checked to ensure topology preservation.

The directional and the subfield strategy share a common drawback: depending on the order of the considered directions or subfields, one can obtain different skeletons. An alternative to these strategies consists of deleting points in a symmetric manner. By symmetric, we mean that this operation is invariant by any isometry (an isometry, in $\mathbb{Z}^{d}$, is a bijection which preserves adjacency relations). For topology preservation, additional conditions must be verified when deleting simple points in this way. Such conditions are difficult to design: indeed, very few symmetric 3D thinning algorithms have been published [31, 32, 37, 29, 40], and among these, [31] and [32] do not preserve topology (see [27, 28]).

Recently, one of the authors introduced a general framework, called critical kernels [8], that permits to

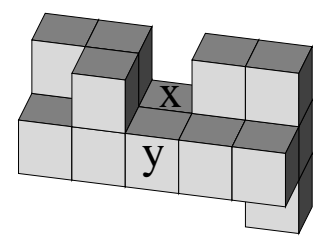

Figure 3: All voxels are simple, the voxels $x$ and $y$ are both "up" voxels. 
check the topological soundness of parallel thinning algorithms in any dimension, and also to design new ones that preserve topology "by construction".

As proven in [12], critical kernels constitute a nontrivial generalization of all previously proposed frameworks with similar aims, namely minimal non-simple sets [42] and P-simple points [6]. Thanks to critical kernels, we were able to propose in [11] nine new 2D thinning algorithms which respond to specific needs (symmetry, centering, thinness, geometrical criterions, etc.) and which had no equivalent among previously published works. The clear separation of topological and geometrical constraints, which is a key feature of this framework, makes easy the design of such algorithms.

The main contribution of the present article consists of new $3 \mathrm{D}$ parallel and symmetric thinning schemes which have the following qualities:

- They are effective and sound, as the main theorem of critical kernels and additional properties proven in this article provide the guarantee of topology preservation;

- They are powerful, in the sense that they remove more points, in one iteration, than any other symmetric parallel thinning scheme. In particular, they can be used to compute minimal skeletons;

- They are versatile, as conditions for the preservation of geometrical features (e.g., curve extremities or surface borders) are independent of those accounting for topology preservation. We give in this article examples and illustrations of minimal, curvilinear and surface skeletons produced using these schemes;

- They are efficient: we provide in this article a small set of masks, acting in the grid $\mathbb{Z}^{3}$, that is sufficient, in addition to the classical simple point test, to straightforwardly implement them.

All the proofs of properties stated below are in the appendix. Some preliminary results of the work presented in this paper appear in [10].

\section{Cubical and Xel Complexes}

In this section, we give some basic definitions for cubical complexes, see also [26, 3, 2]. We consider here the three-dimensional case. Note that most of the notions introduced in the first sections make sense in arbitrary $n$-dimensional cubical spaces.

Let $\mathbb{Z}$ be the set of integers. We consider the families of sets $\mathbb{F}_{0}^{1}, \mathbb{F}_{1}^{1}$, such that $\mathbb{F}_{0}^{1}=\{\{a\} \mid a \in \mathbb{Z}\}, \mathbb{F}_{1}^{1}=$ $\{\{a, a+1\} \mid a \in \mathbb{Z}\}$. A subset $f$ of $\mathbb{Z}^{3}$ which is the Cartesian product of exactly $d$ elements of $\mathbb{F}_{1}^{1}$ and $(n-d)$ elements of $\mathbb{F}_{0}^{1}$ is called a face or a $d$-face of $\mathbb{Z}^{3}, d$ is the dimension of $f$, we write $\operatorname{dim}(f)=d$.

We denote by $\mathbb{F}^{3}$ the set composed of all $d$-faces of $\mathbb{Z}^{3}$, with $d \in\{0,1,2,3\}$. A $d$-face of $\mathbb{Z}^{3}$ is called a point if $d=0$, a (unit) segment if $d=1$, a (unit) square if $d=2$, a (unit) cube if $d=3$.

If $X$ is a finite set of faces in $\mathbb{F}^{3}$, we write $X^{-}=\{y \in$ $\mathbb{F}^{3} \mid y \subseteq x$ for some $\left.x \in X\right\}, X^{-}$is the closure of $X$. A finite set $X$ of faces in $\mathbb{F}^{3}$ is a cubical complex (in $\mathbb{F}^{3}$ ) if $X=X^{-}$. We denote by $\mathbb{C}^{3}$ the collection composed of all such complexes.

Let $X$ be a finite set of faces in $\mathbb{F}^{3}$. We say that $X$ is a xel complex (in $\mathbb{F}^{3}$ ) if, for any $x, y \in X$, we have $y=x$ whenever $y \subseteq x$. We denote by $\mathbb{X}^{3}$ the collection composed of all such complexes. Observe that, if $X \in \mathbb{X}^{3}$ and $Y \subseteq X$, then we have necessarily $Y \in \mathbb{X}^{3}$.

If $X$ is a finite set of faces in $\mathbb{F}^{3}$, we denote by $X^{+}$the set of faces in $X$ which are maximal for inclusion in $X$. Thus, if $X \in \mathbb{C}^{3}$, we have $X^{+} \in \mathbb{X}^{3}$ and $\left(X^{+}\right)^{-}=X$. If $X \in \mathbb{X}^{3}$, we have $X^{-} \in \mathbb{C}^{3}$ and $\left(X^{-}\right)^{+}=X$.

Therefore, it is equivalent, with the above correspondences, to specify a cubical complex or a xel complex. See an illustration Fig. 4.

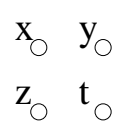

(a)

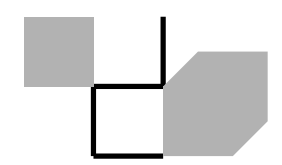

(d)

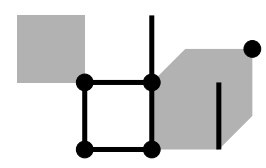

(c)

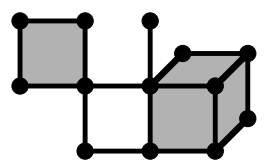

(e)
Figure 4: (a): Four points $x, y, z, t$. (b): A graphical representation of the set of faces $\{\{x, y, z, t\},\{x, y\},\{z\}\}$. (c): A set of faces $X$, which is neither a cubical complex nor a xel complex. (d): The set $X^{+}$, which is a xel complex composed of 4 segments, 1 square, and 1 cube. (e): The set $X^{-}$, which is a cubical complex.

\section{$3 \quad$ Simple Faces}

Intuitively a face $x$ of a xel complex $X$ is simple if its removal from $X$ "does not change the topology of $X$ ". In this section, we propose a definition of a simple face 


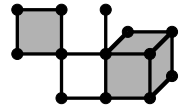

(a)

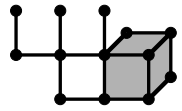

(b)

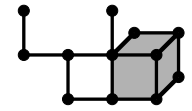

(c)

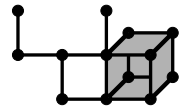

(d)
Figure 5: The cubical complex of Fig. 4 (e) and three steps of elementary collapses.

based on the operation of collapse $[47,19]$. This operation, which is a discrete analogue of a continuous deformation (a homotopy), is defined hereafter for an arbitrary cubical complex $X \in \mathbb{C}^{3}$.

Let $X \in \mathbb{C}^{3}$ and $x, y \in X$ such that $x \subset y$. If $y$ is the only face of $X$ distinct from $x$ that contains $x$, we say that $(x, y)$ is a free pair for $X$, and that the cubical complex $X \backslash\{x, y\}$ is an elementary collapse of $X$.

Let $X, Y \in \mathbb{C}^{3}$. We say that $X$ collapses onto $Y$ if there exists a sequence $\left\langle X_{0}, \ldots, X_{k}\right\rangle$ such that $X_{0}=X$, $X_{k}=Y$, and $X_{i}$ is an elementary collapse of $X_{i-1}, i=$ $1, \ldots, k$. See illustration Fig. 5 .

Now, we give the definition of a simple face in an arbitrary xel complex $X \in \mathbb{X}^{3}$, see [8]. It may be seen as a discrete analogue of the one given by T.Y. Kong in [23] which lies on continuous deformations in the Euclidean space. See the illustration given Fig. 6 .

Definition 1. Let $X \in \mathbb{X}^{3}$ and let $x \in X$. We say that $x$ is simple for $X$ if $X^{-}$collapses onto $(X \backslash\{x\})^{-}$. If $x$ is simple for $X$, we say that $X \backslash\{x\}$ is an elementary thinning of $X$.

Let $X, Y \in \mathbb{X}^{3}$. We say that $Y$ is a thinning of $X$ if there exists a sequence $\left\langle X_{0}, \ldots, X_{k}\right\rangle$ such that $X_{0}=X$, $X_{k}=Y$, and $X_{i}$ is an elementary thinning of $X_{i-1}$, $i=1, \ldots, k$.

Observe that, if $Y$ is a thinning of $X$, then $X^{-}$collapses onto $Y^{-}$.

\section{Critical Kernels}

Let $X$ be a xel complex in $\mathbb{F}^{3}$. As seen in the introduction, if we remove simultaneously (in parallel) simple faces from $X$, we may "change the topology" of the original object $X$. More precisely, we may obtain a set $Y$ such that $X^{-}$does not collapse onto $Y^{-}$.

Thus, it is not possible to use directly the notion of simple face for thinning discrete objects in a symmetrical manner.

In this section, we recall a framework for thinning discrete objects in parallel with the warranty that we do
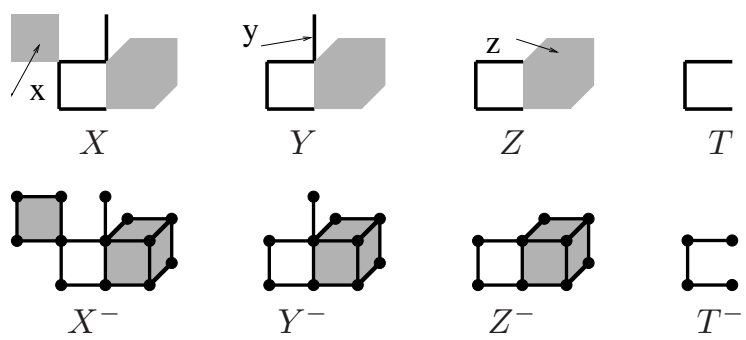

Figure 6: Four xel complexes $X, Y=X \backslash\{x\}, Z=$ $Y \backslash\{y\}, T=Z \backslash\{z\}$ ( $X$ is the xel complex of Fig. 4 (d)). The cubical complexes $X^{-}, Y^{-}, Z^{-}, T^{-}$are also given. The face $x$ is simple for $X, y$ is simple for $Y$, but $z$ is not simple for $Z$, for $Z^{-}$does not collapse on $T^{-}$.

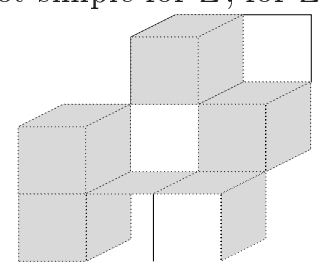

(a)

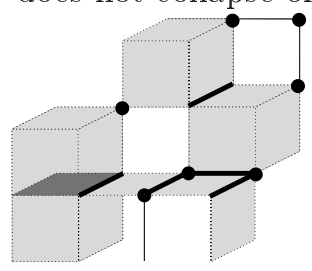

(b)
Figure 7: (a) A xel complex $X$ which is made of 3 segments, 3 squares, and 4 cubes, (b) the faces which are essential for $X$ and which are not faces of $X$ are highlighted in dark.

not alter the topology of these objects [8]. This method holds for complexes of arbitrary dimension. As far as we know, this is the first general method which permits to thin arbitrary complexes in a symmetric way.

Let $C \in \mathbb{X}^{3}$. We say that $C$ is a $d$-clique, or a clique, if $\cap\{x \in C\}$ is a $d$-face.

Definition 2. Let $X \in \mathbb{X}^{3}$ and let $x \in X^{-}$. We say that $x$ is an essential face for $X$ if $x$ is precisely the intersection of all faces of $X$ which contain $x$, i.e., if $x=\cap\{y \in X \mid x \subseteq y\}$. If $x$ is an essential face for $X$, we write $x_{X}^{+}=\{y \in X \mid x \subseteq y\}$, and we say that the clique $x_{X}^{+}$is essential for $X$.

Let $x$ be any face of $X \in \mathbb{X}^{3}$. We observe that $x$ is an essential face for $X$ and we have $x_{X}^{+}=\{x\}$. The essential faces for the xel complex $X$ of Fig. 7 (a) which are not faces of $X$ are highlighted Fig. 7 (b).

Definition 3. Let $X \in \mathbb{X}^{3}$ and let $x$ be an essential face for $X$. We say that $x$ is regular for $X$ if $x$ is simple for $\left(X \backslash x_{X}^{+}\right) \cup\{x\}$. We say that $x$ is critical for $X$ if $x$ is not regular for $X$. If $x$ is critical (resp. regular) for $X$, we say that the clique $x_{X}^{+}$is critical (resp. regular) for $X$. 
Observe that, in the previous definition, $\left(X \backslash x_{X}^{+}\right) \cup\{x\}$ is a xel complex. If $x \in X$, we have $\left(X \backslash x_{X}^{+}\right) \cup\{x\}=X$. Thus, a face $x \in X$ is regular for $X$ if and only if it is simple for $X$. Observe also that a 0 -clique which is essential for $X$ is necessarily critical for $X$. See Fig. 8 and 9 which illustrate the notion of a critical face. Note that an alternative and equivalent definition of a regular/critical face is given in [8].

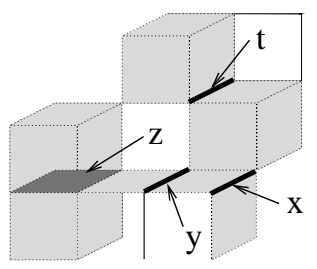

(a)

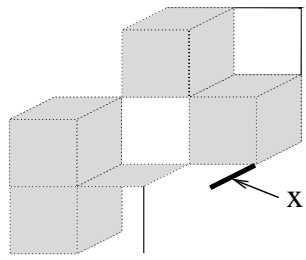

(b)

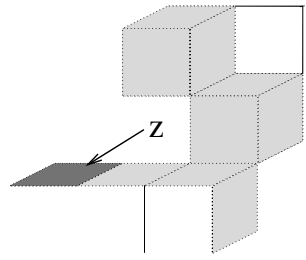

(d)

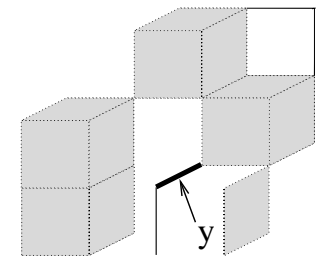

(c)

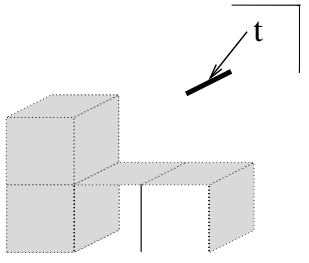

(e)
Figure 8: (a): The xel complex $X$ of Fig. 7 and four essential faces $x, y, z, t$ (highlighted). (b): The xel complex $\left(X \backslash x_{X}^{+}\right) \cup\{x\}: x$ is regular for $X$. (c) The xel complex $\left(X \backslash y_{X}^{+}\right) \cup\{y\}: y$ is critical for $X$. (d): The xel complex $\left(X \backslash z_{X}^{+}\right) \cup\{z\}: z$ is regular for $X$. (e) The xel complex $\left(X \backslash t_{X}^{+}\right) \cup\{t\}: t$ is critical for $X$.

Remark 4. Let $X \in \mathbb{X}^{3}$, let $x$ be an essential face for $X$, and let $C$ be the clique $x_{X}^{+}$. If $C$ is regular for $X$, and if $x \in X$, then (as mentioned above) $x$ is simple for $X$, and we have $C=\{x\}$. Thus $X \backslash C$ is a thinning of $X$ : we can remove such a regular clique from the object without altering the topology. Now let us consider the case where $C$ is regular but $x \notin X$. For that purpose, let

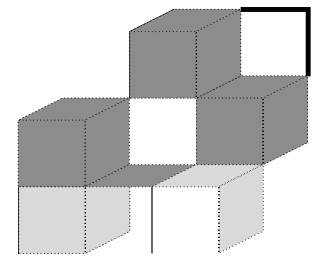

(a)

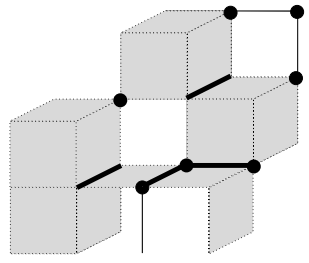

(b)
Figure 9: (a): The xel complex $X$ of Fig. 7: the faces of $X$ which are critical for $X$ (not simple) are highlighted. (b): The faces which are critical for $X$ and which are not faces of $X$ are highlighted.

us consider the xel complex $X$ of Fig. 8 (a) and the faces $x$ and $z$. Let $C$ be the clique (made of 2 squares) such that $C=x_{X}^{+}, C$ is a regular clique for $X$. We observe that $X \backslash C$ is a thinning of $X$ (see Fig. 8 (b)). Now let $C^{\prime}=z_{X}^{+}$(a clique composed of two cubes) which is also a regular clique for $X$. We note that $X \backslash C^{\prime}$ has not the same topology as $X$ ( $X$ has two tunnels and $X \backslash C^{\prime}$ has only one tunnel, see Fig. 8 (a) and (d)). Thus $X \backslash C^{\prime}$ cannot be a thinning of $X$. In fact, the difference between these two situations is that the two faces of $x_{X}^{+}$ are regular (i.e. simple) for $X$, while there is a face of $z_{X}^{+}$which is not regular for $X$ (the cube above $z$ ). In the sequel of this section, we will give some conditions which, in the context of critical faces and critical cliques, ensure that a given subset $Y \subseteq X$ is a thinning of $X$.

The following result is a consequence of a general theorem which holds for complexes of arbitrary dimensions (see [8]).

If $X \in \mathbb{X}^{3}$, the critical kernel of $X$ is the cubical complex composed of all faces that are critical for $X$ and all faces that are included in these faces.

Theorem 5. Let $X \in \mathbb{X}^{3}$ and let $Y \subseteq X$.

The xel complex $Y$ is a thinning of $X$ if $Y^{-}$contains the critical kernel of $X$.

In other words, the xel complex $Y$ is a thinning of $X$ if $Y^{-}$contains all faces that are critical for $X$. See Fig. 10 which provides two examples of a complex $Y$ that satisfies the above property.

As a direct consequence of Th. 5, we obtain the following property which will be our guideline for the sequel. 
Corollary 6. Let $X \in \mathbb{X}^{3}$ and let $Y \subseteq X$.

The xel complex $Y$ is a thinning of $X$ if any clique that is critical for $X$ contains at least one face of $Y$.

We conclude this section by giving a characterization of the complexes which satisfy the condition of Th. 5 .

Theorem 7. Let $X \in \mathbb{X}^{3}$ and let $Y \subseteq X$. The cubical complex $Y^{-}$contains the critical kernel of $X$ if and only if any $Z$ such that $Y \subseteq Z \subseteq X$ is a thinning of $X$.

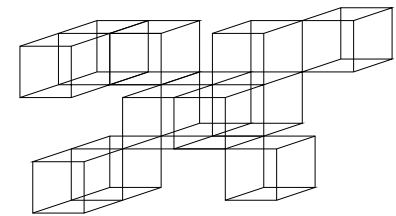

(a)

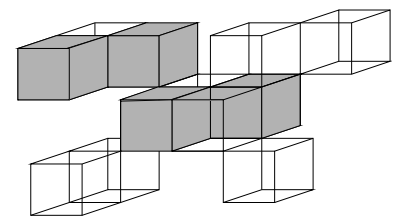

(c)

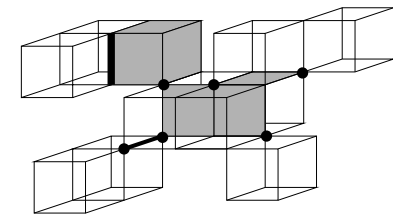

(b)

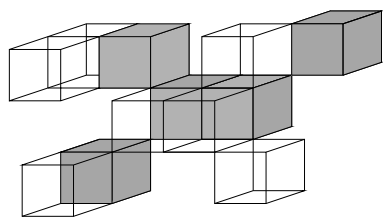

(d)
Figure 10: (a): A xel complex $X$ made of 12 cubes. (b): The faces that are critical for $X$ are highlighted. (c) and (d) : two xel complexes $Y^{\prime} \subseteq X$ and $Y^{\prime \prime} \subseteq X$. By Th. $5, Y^{\prime}$ and $Y^{\prime \prime}$ are both thinnings of $X$.

\section{Characterization of critical cliques in voxel complexes}

In this paper, we investigate a methodology for thinning objects which are made of voxels (i.e., unit cubes). For that purpose, we propose, in the following, several characterizations of $d$-cliques (with $d=3,2,1,0$ ) which are critical for such objects. We first give a few basic definitions for voxel complexes.

We denote by $\mathbb{V}^{3}$ the collection of all xel complexes which are composed solely of unit cubes. A unit cube is also called a voxel, an element of $\mathbb{V}^{3}$ is called a voxel complex.

For example, the xel complex of Fig. 10 (a) is a voxel complex, while the one of Fig. 7 (a) is not.

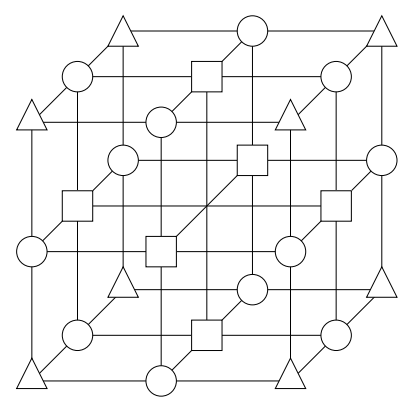

Figure 11: Different types of neighborhoods: $\mathcal{N}_{2}^{*}(x)$ (squares), $\mathcal{N}_{1}^{*}(x)$ (squares and circles), $\mathcal{N}_{0}^{*}(x)$ (squares, circles, and triangles). The voxel $x$ corresponds to the central point.

Let $d \in\{0,1,2\}$. We say that two voxels $x, y$ are $d$-adjacent if $x \cap y$ is a $k$-face, with $k \geq d$. If $x$ is a voxel, we write $\mathcal{N}_{d}(x)$ for the set of all voxels which are $d$-adjacent to $x, \mathcal{N}_{d}(x)$ is the $d$-neighborhood of $x$. Note that, for each voxel $x$, we have $x \in \mathcal{N}_{d}(x)$. We set $\mathcal{N}_{d}^{*}(x)=\mathcal{N}_{d}(x) \backslash\{x\}$. See an illustration Fig. 11 where the voxel $x$ is represented by a point.

Let $X, Y \in \mathbb{V}^{3}$, with $Y \subseteq X$. We say that $Y$ is $d$-connected in $X$ if, for any $x, y \in Y$, there exists a sequence $\left\langle x_{0}, \ldots, x_{k}\right\rangle$ of voxels in $X$ such that $x_{0}=x$, $x_{k}=y$, and $x_{i}$ is $d$-adjacent to $x_{i-1}, i=1, \ldots, k$.

We say that $X \in \mathbb{V}^{3}$ is $d$-connected if $X$ is $d$-connected in $X$.

A 3-clique which is critical for $X \in \mathbb{V}^{3}$ is a set composed solely of one voxel which is not simple for $X$. Thus, any characterization of simple voxels is sufficient to characterize such cliques.

The following proposition shows that, when considering voxel complexes, Definition 1 leads to a characterization of simple voxels which is equivalent to previous ones $[5,13,46,22,17]$. If $X \in \mathbb{V}^{3}$, we write $\bar{X}$ for the set of voxels which are not in $X$.

Proposition 8. Let $X \in \mathbb{V}^{3}$ and let $x \in X$.

The voxel $x$ is simple for $X$ if and only if:

1) The set $\mathcal{N}_{0}^{*}(x) \cap X$ is non-empty and 0 -connected; and

2) The set $\mathcal{N}_{2}^{*}(x) \cap \bar{X}$ is non-empty and 2-connected in $\mathcal{N}_{1}^{*}(x) \cap \bar{X}$.

Let $d \in\{0,1,2\}$. The voxels which belong to $d$-cliques that are critical for $X \in \mathbb{V}^{3}$ may be detected by:

1) Detecting all $d$-faces in $X^{-}$which are essential for $X$ 
(Def. 2);

2) Detecting all essential $d$-faces $x$ which are not simple for $\left(X \backslash x_{X}^{+}\right) \cup\{x\}$ (Def. 3);

3) Labeling all the voxels of $X$ which contain such faces.

In the following, we propose to characterize critical cliques in a way such that the computation of $X^{-}$is not necessary.

We first observe that, up to $\pi / 2$ rotations, the three configurations $\mathcal{C}_{2}, \mathcal{C}_{1}$, and $\mathcal{C}_{0}$ given in Fig. 12 may be used for the detection of an arbitrary (regular or critical) clique which is essential for a given voxel complex $X$ in $\mathbb{V}^{3}$ (in this figure a voxel is represented by a point). In fact, it may be seen that:

- $\mathcal{C}_{2}$ may be used for detecting a 2-clique $C$ which is essential for $X$ : there is such a clique if both voxels $A$ and $B$ are in $X$. In this case, we have $C=\{A, B\}$.

- $\mathcal{C}_{1}$ may be used for detecting a 1-clique $C$ which is essential for $X$ : there is such a clique if both $A$ and $D$ are in $X$ or both $B$ and $C$ are in $X$. In this case, we have $C=\{A, B, C, D\} \cap X$.

- $\mathcal{C}_{0}$ may be used for detecting a 0 -clique $C$ which is essential for $X$ : there is such a clique if $A$ and $H$, or $B$ and $G$, or $C$ and $F$, or $D$ and $E$ are in $X$. In this case, we have $C=\{A, B, C, D, E, F, G, H\} \cap X$.

We now introduce a notion of neighborhood which is fundamental for our purpose.

Definition 9. Let $S \in \mathbb{V}^{3}$. The $\mathcal{K}$-neighborhood of $S$, written $\mathcal{K}(S)$, is the set made of all voxels which are 0 -adjacent to each voxel in $S$. We set $\mathcal{K}^{*}(S)=\mathcal{K}(S) \backslash S$.

We note that we have $\mathcal{K}(S)=\mathcal{N}_{0}(x)$ whenever $S$ is made of a single voxel $x$. We also observe that:

- we have $\mathcal{K}(T) \subseteq \mathcal{K}(S)$ whenever $S \subseteq T$;

- we have $S \subseteq \mathcal{K}(S)$ whenever $S$ is a clique;

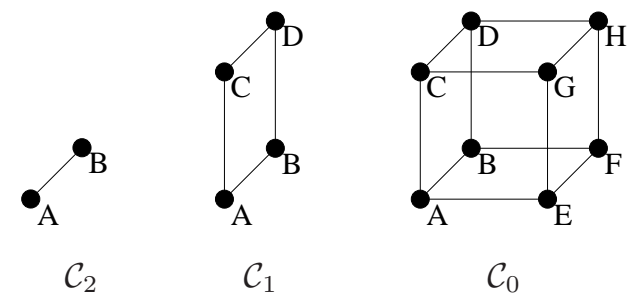

Figure 12: Masks for 2-cliques $\left(\mathcal{C}_{2}\right)$, 1-cliques $\left(\mathcal{C}_{1}\right)$, and 0 -cliques $\left(\mathcal{C}_{0}\right)$. Here, a voxel is represented by its central point.
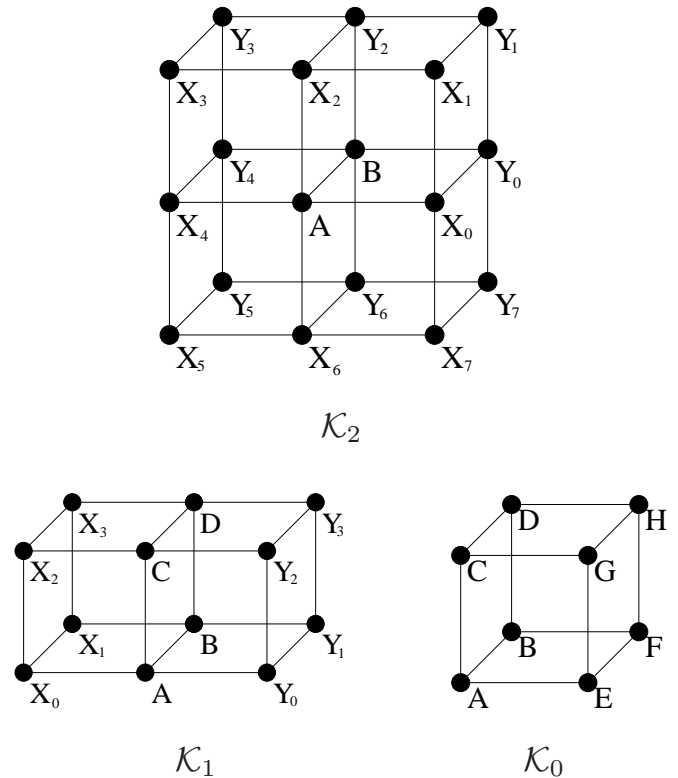

Figure 13: $\mathcal{K}$-neighborhoods for 2-cliques $\left(\mathcal{K}_{2}\right)$, 1-cliques $\left(\mathcal{K}_{1}\right)$, and 0 -cliques $\left(\mathcal{K}_{0}\right)$. A voxel is represented by its central point.

- we have $\mathcal{K}(S)=\mathcal{K}(T)$ whenever $S$ and $T$ are two cliques such that $\cap\{x \in S\}=\cap\{x \in T\}$.

The $\mathcal{K}$-neighborhoods of the configurations $\mathcal{C}_{2}, \mathcal{C}_{1}$, and $\mathcal{C}_{0}$ are given Fig. 13. Observe that we have $\mathcal{K}^{*}(S)=\emptyset$ for the configuration $\mathcal{C}_{0}$.

Let $X \in \mathbb{V}^{3}$. As mentioned earlier, a 0 -clique which is essential for $X$ is necessarily critical. With the following two propositions, we give some characterizations for 2and 1-cliques which are regular for $X$. Recall that a 2clique which is essential for $X$ is necessarily composed of two voxels which are 2 -adjacent (configuration $\mathcal{C}_{2}$ ).

Proposition 10. Let $X \in \mathbb{V}^{3}$, let $C=\{x, y\}$ be a 2clique which is essential for $X$. The clique $C$ is regular for $X$ if and only if:

1) The set of voxels $\mathcal{K}^{*}(C) \cap X$ is non-empty and 0connected; and

2) There exists two voxels $x^{\prime}, y^{\prime} \in \mathcal{K}^{*}(C) \cap \bar{X}$ such that $x^{\prime} \in \mathcal{N}_{2}^{*}(x), y^{\prime} \in \mathcal{N}_{2}^{*}(y)$, and $x^{\prime} \in \mathcal{N}_{2}^{*}\left(y^{\prime}\right)$.

Proposition 11. Let $X \in \mathbb{V}^{3}$, let $C$ be a 1-clique which is essential for $X$. The clique $C$ is regular for $X$ if and only if the set of voxels $\mathcal{K}^{*}(C) \cap X$ is non-empty and 0-connected.

We are now in position to propose some masks for detecting critical cliques. These masks $\mathcal{K}_{2}, \mathcal{K}_{1}, \mathcal{K}_{0}$ are 


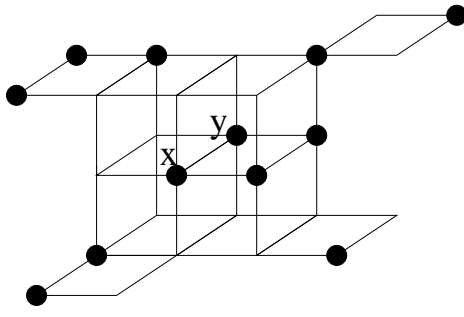

(a)

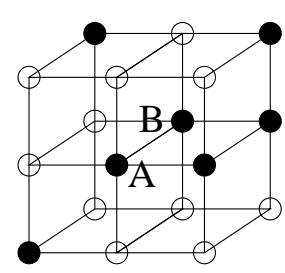

(b)

Figure 14: (a): The xel complex $X$ which is the one of Fig. 10 (a). Here, each voxel of $X$ is represented by a black disk. (b): The mask $\mathcal{K}_{2}$, with $A, B$ matching voxels $x, y$ of $X$. Condition ii) of Def. 12 for $\mathcal{K}_{2}$ is not satisfied but condition $\mathrm{i}$ ) is fulfilled since the set of voxels $\left\{X_{0}, \ldots, X_{7}, Y_{0}, \ldots, Y_{7}\right\} \cap X$ is not 0 -connected. Thus, by Prop. 13, the voxels $x, y$ constitute a 2 -clique of $S$ which is critical for $X$. See also Fig. 10 (b) where the critical face $z=x \cap y$ is highlighted, we have $z_{X}^{+}=\{x, y\}$.

described using Fig. 13. For each of these masks, we also consider all the masks obtained from them by applying $\pi / 2$ rotations about each axis. We get 7 masks $\left(3\right.$ for $\mathcal{K}_{2}$, 3 for $\mathcal{K}_{1}$, and 1 for $\mathcal{K}_{0}$ ). See Fig. 14 for an illustration of the use of the mask $\mathcal{K}_{2}$.

Definition 12. Let $X \in \mathbb{V}^{3}$, and let $S$ be a set of voxels of $X$. We say that:

1) $S$ matches $\mathcal{K}_{2}$ in $X$ if $S=\{A, B\}$; and

i) the set of voxels $\left\{X_{0}, \ldots, X_{7}, Y_{0}, \ldots, Y_{7}\right\} \cap X$ is either empty or not 0 -connected; or

ii) for each $i \in\{0,2,4,6\}, X_{i}$ or $Y_{i}$ belongs to $X$. 2) $S$ matches $\mathcal{K}_{1}$ in $X$ if $S=\{A, B, C, D\} \cap X$; and

i) at least one of the sets $\{A, D\},\{B, C\}$ is a subset of $X$; and

ii) we have either [ $U \cap X \neq \emptyset$ and $V \cap X \neq \emptyset$ ] or [ $U \cap X=\emptyset$ and $V \cap X=\emptyset$ ], with $U=\left\{X_{0}, \ldots, X_{3}\right\}$ and $V=\left\{Y_{0}, \ldots, Y_{3}\right\}$.

3) $S$ matches $\mathcal{K}_{0}$ in $X$ if $S=\{A, B, C, D, E, F, G, H\} \cap X$ and at least one of the sets $\{A, H\},\{B, G\},\{C, F\}$, $\{D, E\}$ is a subset of $X$.

Prop. 13 is a direct consequence of Prop. 10 and 11.

Proposition 13. Let $X \in \mathbb{V}^{3}$, let $S$ be a set of voxels in $X$, and let $d \in\{2,1,0\}$. The set $S$ is a d-clique which is critical for $X$ if and only if $S$ matches $\mathcal{K}_{d}$ in $X$.

We conclude this section by giving a characterization of simple voxels and regular cliques that is based on the

notion of a reducible set of voxels. A reducible set is defined recursively as follows.

Definition 14. Let $X \in \mathbb{V}^{3}$.

We say that $X$ is reducible if either:

i) $X$ is composed of a single voxel; or

ii) there exists $x \in X$ such that $\mathcal{N}_{0}^{*}(x) \cap X$ is reducible and $X \backslash\{x\}$ is reducible.

The following theorem allows us to characterize simple voxels with reducible sets, see also $[23,7]$ for other recursive approaches for simplicity.

Theorem 15. Let $X \in \mathbb{V}^{3}$ and let $x \in X$. The voxel $x$ is simple for $X$ if and only if $\mathcal{N}_{0}^{*}(x) \cap X$ is reducible.

Thus, a complex $X \in \mathbb{V}^{3}$ is reducible if and only if it is possible to reduce $X$ to a single voxel by iteratively removing simple voxels.

More precisely, $X \in \mathbb{V}^{3}$ is reducible if and only if there exists a sequence $\left\langle x_{0}, \ldots, x_{k}\right\rangle$ such that $X=\left\{x_{0}, \ldots, x_{k}\right\}$, and $x_{i}$ is simple for $\left\{x_{i}, \ldots, x_{k}\right\}, i \in[0, k-1]$.

The following theorem is an extension of Th. 15 to arbitrary regular cliques.

Theorem 16. Let $X \in \mathbb{V}^{3}$ and let $C$ be a clique that is essential for $X$. The clique $C$ is regular for $X$ if and only if $\mathcal{K}^{*}(C) \cap X$ is reducible.

Thus, Th. 16 makes it possible to characterize, in a unified way, regular $d$-cliques, with $d=3,2,1,0$. In particular, for $d=3$, we get the characterization of simple voxels given Th. 15 . In this case, the clique $C$ is made of a single voxel $x$, and we have $\mathcal{K}^{*}(C)=\mathcal{N}_{0}^{*}(x)$.

Let $X \in \mathbb{V}^{3}$ be a reducible complex which is not composed of a single voxel. By the very definition of such a complex, and by Th. 15, there exists a simple voxel for $X$ such that $X \backslash\{x\}$ is reducible. But if $x$ is an arbitrary simple voxel for $X$, then $X \backslash\{x\}$ is not necessarily reducible. Such a situation occurs when $X \backslash\{x\}$ is an object such as the so-called dunce hat [48] or house with two rooms [14], see also [34] for algorithmic issues.

The following result shows that there is not enough space for such objects to be in the $\mathcal{K}$-neighborhood of a clique.

Theorem 17. Let $C \in \mathbb{V}^{3}$ such that $C$ is a clique, and let $S \subseteq \mathcal{K}^{*}(C)$. If $S$ is reducible, then $S \backslash\{x\}$ is reducible whenever $x$ is a simple voxel for $S$. 
Let $X \in \mathbb{V}^{3}$ and let $C$ be a clique that is essential for $X$. As a consequence of Th. 16 and 17, determining whether $C$ is regular or critical for $X$ may be done by the following greedy algorithm RegularClique.

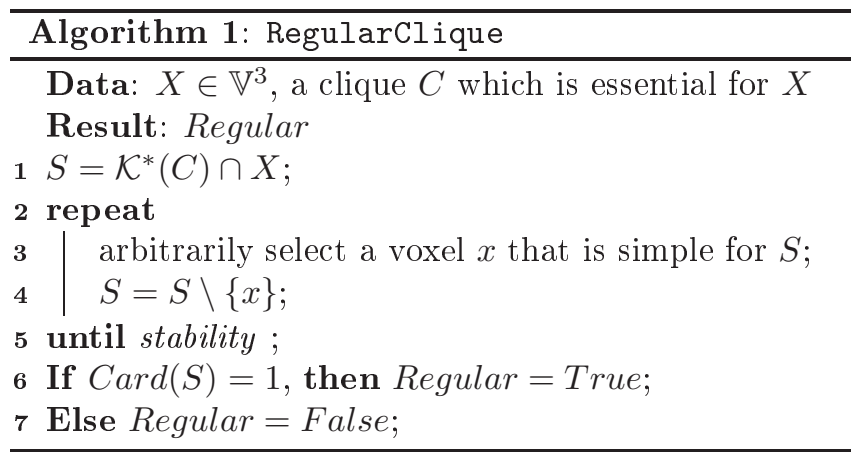

\section{Crucial Kernels and Minimal Skeletons}

Our goal is to define a subset of a voxel complex $X$ that is guaranteed to include at least one voxel of each clique that is critical for $X$. By Cor. 6, this subset will be a thinning of $X$.

We want this subset to be as small as possible in order to obtain an efficient thinning procedure. We also want our method to be independent of arbitrary choices, in particular of a choice of specific voxels in a given critical clique. For that purpose the following notion of a "maximal critical face" was introduced [11, 12].

Let $X \in \mathbb{V}^{3}$ and let $x$ be a critical face for $X$. We say that $x$ is $\mathcal{M}$-critical for $X$ if $x$ is not a proper face of a face which is critical for $X$. If $x$ is $\mathcal{M}$-critical for $X$, we say that the clique $x_{X}^{+}$is $\mathcal{M}$-crucial for $X$. We say that a voxel $x \in X$ is $\mathcal{M}$-crucial for $X$ if $x$ belongs to a clique which is $\mathcal{M}$-crucial for $X$.

If $X \in \mathbb{V}^{3}$, we denote by $\mathcal{M}(X)$ the set composed of all voxels that are $\mathcal{M}$-crucial for $X, \mathcal{M}(X)$ is the $\mathcal{M}$-crucial kernel of $X$. Thus, $\mathcal{M}(X)$ is the set of voxels of $X$ that contain a face which is $\mathcal{M}$-critical for $X$.

In Fig. 15 (a), the $\mathcal{M}$-critical faces of a complex $X$ are highlighted (see also Fig. 10 (b) where the critical faces of the same complex are given). The $\mathcal{M}$-crucial kernel of $X$ is given Fig. 15 (b).
Remark 18. Let $X \in \mathbb{V}^{3}$ and let $C \subseteq X$. It may be seen that $C$ is an $\mathcal{M}$-crucial clique for $X$ if and only if $C$ is a critical clique for $X$ and no proper subset of $C$ is a clique which is critical for $X$.

Remark 19. Let $X \in \mathbb{V}^{3}$ and let $C \subseteq X$. It has been proved that $C$ is non-simple for $X$ whenever $C$ is an $\mathcal{M}$-crucial clique for $X$, which means that the set $X \backslash C$ is not a thinning of $X$. In fact, it was shown in [12] (Th. 28), that a subset of $X$ is an $\mathcal{M}$-crucial clique for $X$ if and only if it is a minimal non-simple set for $X$, see $[42,21,30,24]$ for other properties of the so-called MNS's.

By the very definition of an $\mathcal{M}$-crucial voxel, $\mathcal{M}(X)$ is guaranteed to include at least one voxel of each clique which is critical for $X$, thus $\mathcal{M}(X)$ is a thinning of $X$. Nevertheless, through the following observation, it may be seen that it is possible to obtain a subset of voxels of $X$ which fulfills the conditions given in the very beginning of this section, and which contains less voxels than $\mathcal{M}(X)$.

Let us consider again Fig. 15 (a). The voxel $x$ contains an $\mathcal{M}$-critical 1 -face and thus it belongs to $\mathcal{M}(X)$. But this 1-face is also included in the voxel $y$, which contains a 2 -face which is also $\mathcal{M}$-critical. This motivates the following recursive definition of a crucial voxel which is based on dimension.

Definition 20. Let $X \in \mathbb{V}^{3}$ and $C$ be a $d$-clique which is critical for $X$. We say that $C$ is $\mathcal{D}$-crucial for $X$ if:

i) $d=3$; or

ii) $d \in\{2,1,0\}$ and $C$ does not contain any voxel belonging to a $d^{\prime}$-clique that is $\mathcal{D}$-crucial for $X$ and such that $d^{\prime}>d$.

We say that a voxel $x \in X$ is $\mathcal{D}$-crucial for $X$ if $x$ belongs to a clique that is $\mathcal{D}$-crucial for $X$.

Note that a voxel that is not simple necessarily constitutes a 3 -clique which is $\mathcal{D}$-crucial. Observe also that, if $d^{\prime} \neq d$, a voxel $x$ that belongs to a $d$-clique which is $\mathcal{D}$-crucial cannot belong to a $d^{\prime}$-clique which is also $\mathcal{D}$-crucial.

If $X \in \mathbb{V}^{3}$, we denote by $\mathcal{D}(X)$ the set composed of all voxels which are $\mathcal{D}$-crucial for $X, \mathcal{D}(X)$ is the $\mathcal{D}$-crucial kernel of $X$.

Again, by the very definition of a $\mathcal{D}$-crucial face, $\mathcal{D}(X)$ is guaranteed to include at least one voxel of each clique which is critical for $X$. Thus, by Cor. $6, \mathcal{D}(X)$ is a thinning of $X$. 
The following proposition shows that, in the context of thinning, the $\mathcal{D}$-crucial kernel corresponds to an operation which is "more powerful" than the $\mathcal{M}$-crucial kernel.

Theorem 21. Let $X \in \mathbb{V}^{3}$. The $\mathcal{D}$-crucial kernel of $X$ is a subset of its $\mathcal{M}$-crucial kernel.

Observe that the example of Fig. 15 (a) shows that the above inclusion may be strict: the voxel $x$ is $\mathcal{M}$-crucial for $X$ but not $\mathcal{D}$-crucial for $X$.

Let $X \in \mathbb{V}^{3}$. Let $\left\langle X_{0}, \ldots, X_{k}\right\rangle$ be the sequence of distinct elements such that $X_{0}=X, X_{k}=\mathcal{D}\left(X_{k}\right)$, and $X_{i}=\mathcal{D}\left(X_{i-1}\right)$, for $i=1, \ldots, k$. The set $X_{k}$ is the minimal $\mathcal{D}$-skeleton of $X$.

In Fig. 15 (c), the complex $Z=\mathcal{D}(X)$ is highlighted, the complex $X$ being the one of Fig. 15 (a). The complex $Z^{\prime}=\mathcal{D}(Z)$ is given in (d) and (e). We have $Z^{\prime}=\mathcal{D}\left(Z^{\prime}\right)$, thus $Z^{\prime}$ is the minimal $\mathcal{D}$-skeleton of $X$.

Two other examples of minimal $\mathcal{D}$-skeletons are given Fig. 16. We will see in the next section that a minimal $\mathcal{D}$-skeleton may be obtained by an algorithm which is a special instance of a generic parallel thinning scheme.

\section{Three Generic Symmetric Thin- ning Schemes}

In this section, we propose three generic thinning schemes which permit to compute a wide variety of skeletons.

For that purpose, we first introduce the notion of a $\mathcal{D}$ crucial kernel which is constrained to preserve a given set $K$ (Def. 23), and which generalizes the definition of a $\mathcal{D}$ crucial kernel presented in Sec. 6. In fact, for thinning objects, we often want to keep other voxels than the ones that are crucial. Intuitively, the set $K$ corresponds to a set of features that we want to be preserved by a thinning algorithm (like extremities of curves, if we want to obtain a curvilinear skeleton).

All the three proposed thinning schemes are based on such constrained $\mathcal{D}$-crucial kernels.

Definition 22. Let $X \in \mathbb{V}^{3}, K \in \mathbb{V}^{3}$, and let $C$ be a $d$-clique which is critical for $X$ and such that $C \subseteq X \backslash K$. We say that $C$ is $\mathcal{D}$-crucial for $\langle X, K\rangle$ if:

i) $d=3$; or

ii) $d \in\{2,1,0\}$ and $C$ does not contain any voxel belonging to a $d^{\prime}$-clique which is $\mathcal{D}$-crucial for $\langle X, K\rangle$ and such that $d^{\prime}>d$.

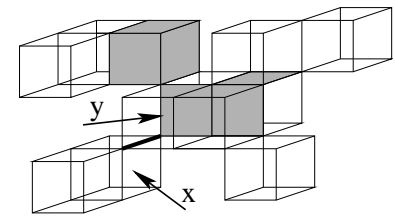

(a)

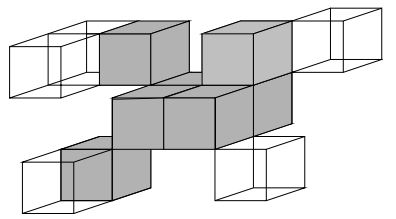

(b)

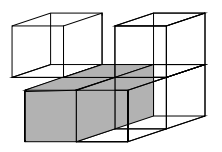

(d)

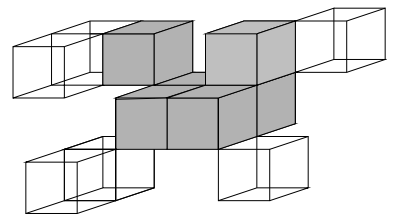

(c)
Figure 15: (a): A voxel complex $X$ and its $\mathcal{M}$-critical faces (highlighted). (b): The complex $Y=\mathcal{M}(X)$ is highlighted. (c): The complex $Z=\mathcal{D}(X)$ is highlighted. (d): The complex $Z^{\prime}=\mathcal{D}(Z)$ is highlighted. (e): We have $Z^{\prime}=\mathcal{D}\left(Z^{\prime}\right)$ : $Z^{\prime}$ is the minimal $\mathcal{D}$-skeleton of $X$.

We say that a voxel $x \in X$ is $\mathcal{D}$-crucial for $\langle X, K\rangle$ if $x$ is in $K$ or if $x$ belongs to a clique which is $\mathcal{D}$-crucial for $\langle X, K\rangle$.

Definition 23. Let $X \in \mathbb{V}^{3}, K \in \mathbb{V}^{3}$. We denote by $\mathcal{D}(X, K)$ the set composed of all voxels which are $\mathcal{D}$ crucial for $\langle X, K\rangle, \mathcal{D}(X, K)$ is the $\mathcal{D}$-crucial kernel of $X$ constrained by $K$.

From the previous definitions and from Cor. 6, we immediately deduce the following proposition which ensures that any constrained $\mathcal{D}$-crucial kernel of an object preserves the topology of this object.

Proposition 24. Let $X \in \mathbb{V}^{3}, K \in \mathbb{V}^{3}$. The $\mathcal{D}$-crucial kernel of $X$ constrained by $K$ is a thinning of $X$.

By construction, the following procedure $\mathrm{D}$-crucial computes the $\mathcal{D}$-crucial kernel of an object $X \in \mathbb{V}^{3}$ constrained by $K$. It consists of 5 steps, each step may be done in parallel. Voxels that are not simple and critical cliques may be detected with the characterizations given Prop. 8 and Prop. 13, or with the 


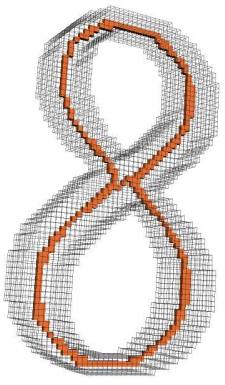

(a)

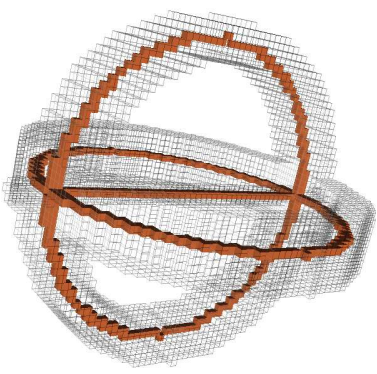

(b)
Figure 16: Two voxel complexes and their minimal $\mathcal{D}$ skeleton (in red).

unified characterization given Th. 16 which can be implemented using algorithm RegularClique.

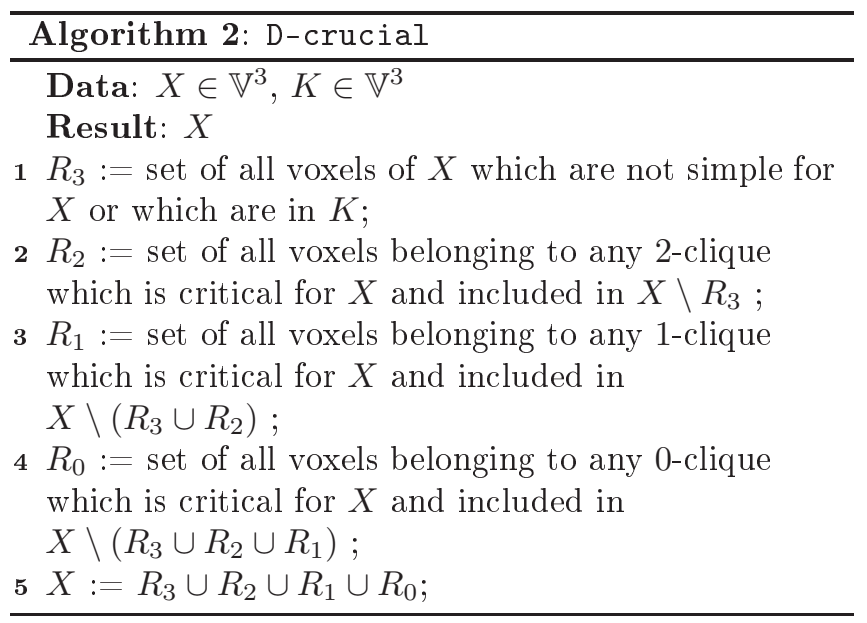

We present now the first thinning scheme which consists in computing iteratively, starting from $X, \mathcal{D}$-crucial kernels constrained by a given set $K$, this constraint set is fixed from the beginning. By Prop. 24, the result is a thinning of $X$. Furthermore, the result contains $K \cap X$.

Definition 25. Let $X \in \mathbb{V}^{3}, K \in \mathbb{V}^{3}$. Let $\left\langle X_{0}, \ldots, X_{k}\right\rangle$ be the sequence of distinct elements such that $X_{0}=X$, $X_{i}=\mathcal{D}\left(X_{i-1}, K\right)$ for $i=1, \ldots, k$, and $X_{k}=\mathcal{D}\left(X_{k}, K\right)$. The set $X_{k}$ is the $\mathcal{D}$-skeleton of $X$ constrained by $K$.

Observe that the minimal $\mathcal{D}$-skeleton of an object $S$ is a $\mathcal{D}$-skeleton of $S$ constrained by $K$, with $K=\emptyset$.

Note also that the $\mathcal{D}$-skeleton of $X$ constrained by $K$ may be easily obtained by repeating, until stability, the procedure D-crucial.

The second thinning scheme is based on a "dynamic constraint set". This constraint set is defined thanks to a function $\Psi$ from $\mathbb{V}^{3}$ to $\mathbb{V}^{3}$ which is fixed from the beginning. This function allows one to define, at each iteration, the very subset of the object which must be preserved during the thinning procedure. Again, by Prop. 24 , the result is a thinning of $X$.

Definition 26. Let $\Psi$ be a function from $\mathbb{V}^{3}$ to $\mathbb{V}^{3}$. Let $X \in \mathbb{V}^{3}$ and let $\left\langle X_{0}, \ldots, X_{k}\right\rangle$ be the sequence of distinct elements such that $X_{0}=X, X_{i}=\mathcal{D}\left(X_{i-1}, \Psi\left(X_{i-1}\right)\right)$ for $i=1, \ldots, k$, and $X_{k}=\mathcal{D}\left(X_{k}, \Psi\left(X_{k}\right)\right)$. The set $X_{k}$ is the $\mathcal{D}$-skeleton of $X$ constrained by $\Psi$.

The third thinning scheme is based, as above, on a dynamic constraint set and a map $\Psi$ from $\mathbb{V}^{3}$ to $\mathbb{V}^{3}$ which is fixed from the beginning. The difference is that the constraint set is built iteratively from $\Psi$ and from the constraint obtained at the previous iteration step. By Prop. 24, the result is a thinning of $X$.

Definition 27. Let $\Psi$ be a function from $\mathbb{V}^{3}$ to $\mathbb{V}^{3}$. Let $X \in \mathbb{V}^{3}, K \in \mathbb{V}^{3}$, and let $\left\langle X_{0}, \ldots, X_{k}\right\rangle$ be the sequence of distinct elements such that $X_{0}=X, K_{0}=K, X_{i}=$ $\mathcal{D}\left(X_{i-1}, K_{i}\right)$ with $K_{i}=K_{i-1} \cup \Psi\left(X_{i-1}\right)$ for $i=1, \ldots, k$, and $X_{k}=\mathcal{D}\left(X_{k}, K_{k} \cup \Psi\left(X_{k}\right)\right)$. The set $S_{k}$ is the $\mathcal{D}$ skeleton of $X$ incrementally constrained by $\Psi$ and $K$.

Again, it may be seen that the $\mathcal{D}$-skeleton of $X$ constrained by $\Psi$, or the $\mathcal{D}$-skeleton of $X$ incrementally constrained by $\Psi$ and $K$, may be easily obtained by iteratively applying the procedure $\mathrm{D}$-crucial.

\section{Examples}

In this section, we give several examples of specific instances of the three above thinning schemes.

A first basic example of a $\mathcal{D}$-skeleton constrained by a set of voxels $K$ is given Fig. 17. Here $K$ is made of 5 points, thus the $\mathcal{D}$-skeleton of the original object $X$ constrained by $K$ is a curvilinear shape. Note that a $\mathcal{D}$-skeleton may contain some simple points that do not belong to the constrained set. In other words such a skeleton may be "thick", which is the price to pay for symmetry.

A second example of such a skeleton is given Fig. 18 where $X$ is a solid cube and $K$ is a subset of $X$ which 
is a solid torus. The $\mathcal{D}$-skeleton of $X$ constrained by $K$ is an object which contains the torus and which has no hole. Since the topology of the cube is preserved during the thinning process, we see that this method may be used for closing the holes of all objects provided they are connected. See also [1].

The quality of a surface skeleton is often assessed by the fact that it contains, approximately or completely, the medial axis of the shape. An easy way to obtain such a skeleton of an object $X$ is to compute the $\mathcal{D}$-skeleton of $X$ constrained by its medial axis $\mathrm{MA}(X)$, the medial axis of $X$ being made of all the centers of the maximal balls included in $X$. Recall that a ball is maximal for $X$ if it is included in $X$ and if it is not a proper subset of another ball included in $X[41,15]$. See Fig. 19 and 20 where an example of a $\mathcal{D}$-skeleton constrained by the medial axis is given, here the city-block distance is considered for defining the balls involved in the medial axis. It is well-known that, with the city-block distance, the medial axis of a shape can be obtained by detecting the local maxima of its distance transform [45]. This provides an efficient algorithm for computing the set $\operatorname{MA}(X)$.

This strategy is effective in $2 \mathrm{D}$, because the existence of a single medial axis point is sufficient to generate a skeleton branch, even in the case where the medial axis is disconnected. However in 3D, the medial axis of certain surface-like objects may be quite sparse, and the skeleton constrained by this medial axis may present unwanted indentations (see the top of Fig. 20).

In order to obtain surface skeletons which do not present such indentations, we may constrain the skeletons with residual voxels. Following the vocabulary introduced in [11], we say that a voxel $x$ in $X$ is a residual voxel (for $X$ ) if it is a border voxel of $X$ which is not 2adjacent to any interior voxel of $X$. Here a border (resp. interior) voxel of $X$ is a voxel of $X$ which is (resp. which is not) 2-adjacent to a voxel in $\bar{X}$. Intuitively, a residual voxel can be located at curvilinear or surface parts of the object.

Let $\Psi$ be the map which associates to $X$ the set composed of all residual voxels for $X$. The $\mathcal{D}$-skeleton of $X$ constrained by $\Psi$ is depicted Fig. 21, $X$ being the object of Fig. 19. We observe that no more "indentations" such as the ones of Fig. 20 appear.

As in 2D (see [11]), this strategy produces a skeleton which contains most of the medial axis points, but not necessarily all of them. For instance, the skeleton shown
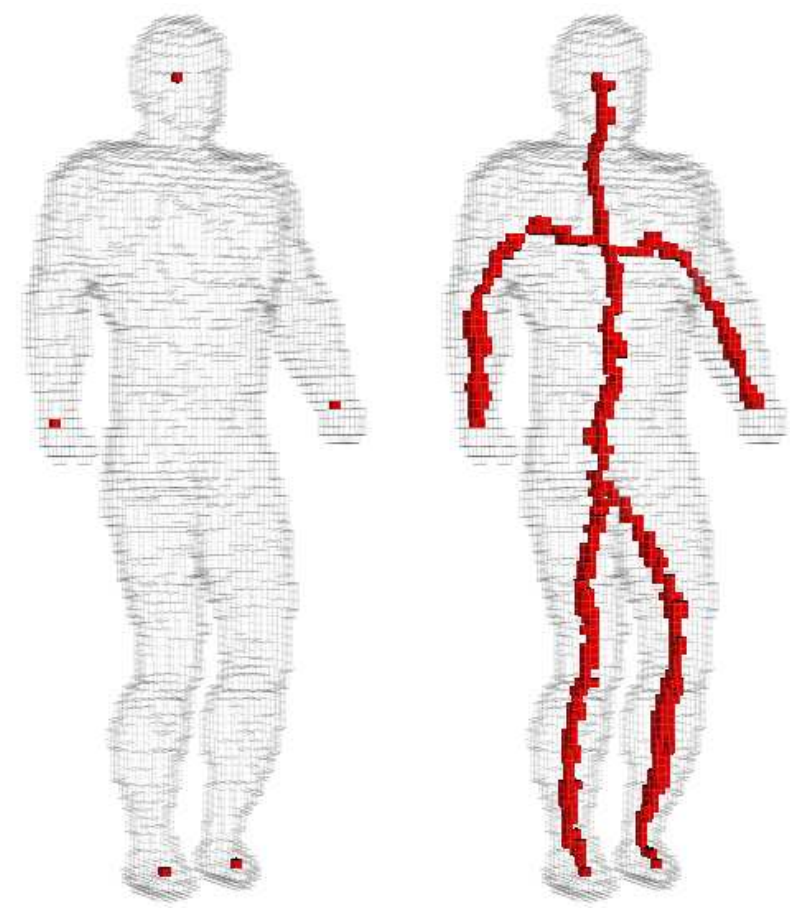

Figure 17: Left: in transparent gray, a shape $X$; in red, five points that will serve as a constraint set $K$. Right: the $\mathcal{D}$-skeleton of $X$ constrained by $K$.
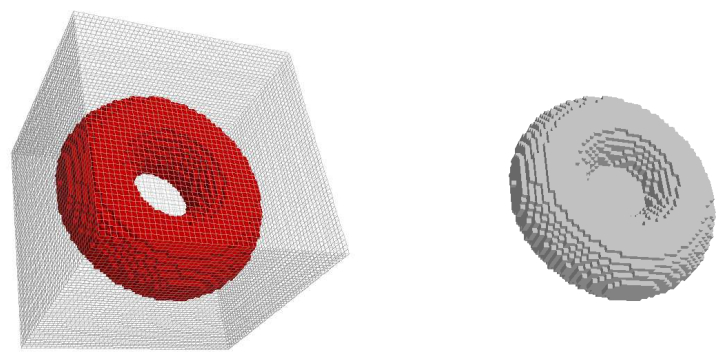

Figure 18: Left: in transparent gray, an object $X$ which is a solid cube; in red, a subset $K$ of $X$ which is a solid torus. Right: the $\mathcal{D}$-skeleton of $X$ constrained by $K$. Notice that the hole of the torus has been closed.

in Fig. 21 contains 1987 among the 1995 medial axis points (only 8 are missing).

A possible way to keep the good quality of the skeleton based on residual voxels while preserving all points of the medial axis, is to consider the $\mathcal{D}$-skeleton of $X$ 

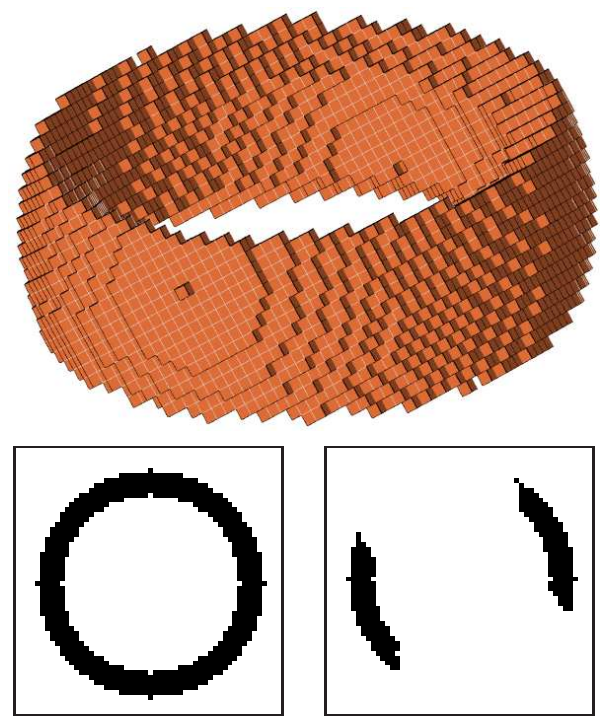

Figure 19: Original object $X$. Up: a rendering (projection). Down: two cross-sections.
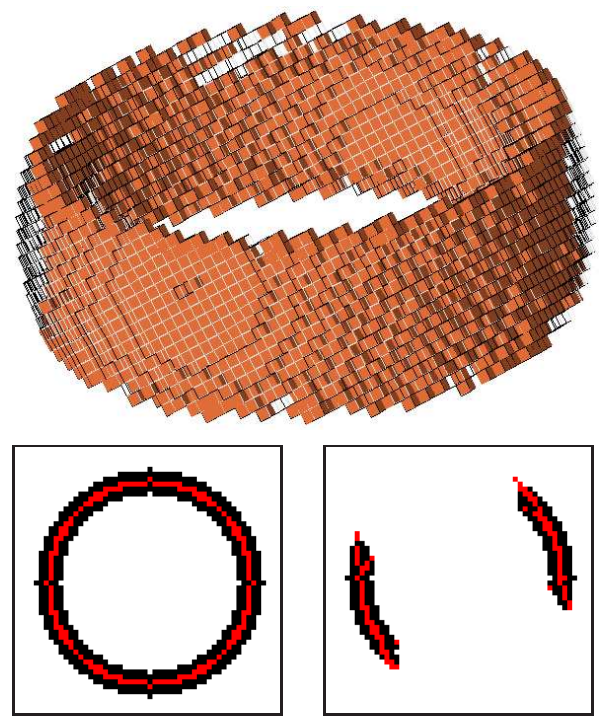

Figure 20: The $\mathcal{D}$-skeleton of $X$ (the object of Fig. 19) constrained by its medial axis.

incrementally constrained by $\Psi$ and $K$. We set the map $\Psi$ to be the above map which associates residual voxels and $K$ to be the medial axis of the original object $X$. When applied to the object of Fig. 19, this skeleton differs only in few voxels with the one of Fig. 21.
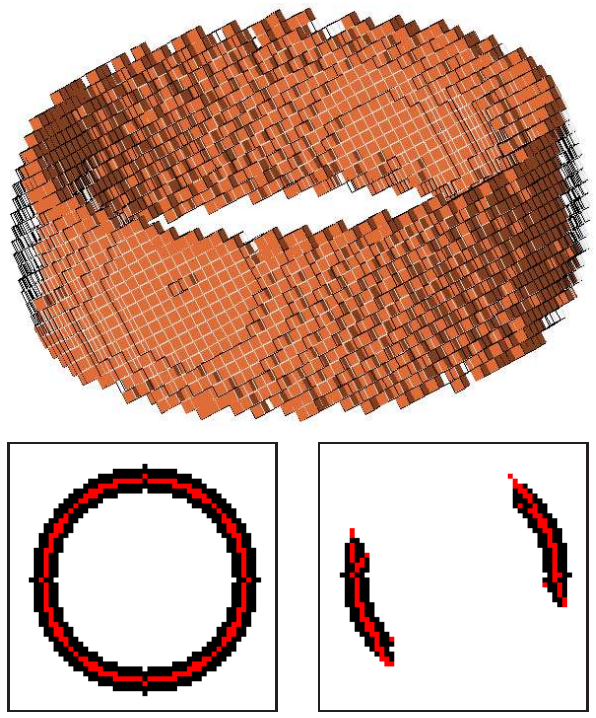

Figure 21: The $\mathcal{D}$-skeleton of $X$ (the object of Fig. 19) constrained by $\Psi$, where $\Psi$ is the map which associates to $X$ the set composed of all residual voxels for $X$.

\section{Discussion and conclusion}

We introduced in this paper new general 3D parallel thinning schemes which are symmetric (invariant by isometries), well defined (which provide results which do not depend on any arbitrary choice), effective and sound (thanks to the properties proved in the critical kernels framework), versatile (allows the user to specify any additional geometrical condition), simple to implement and efficient (they can be implemented through a set of only three masks in addition to the classical simple point test). No previously proposed method exhibits all these qualities. In particular, formerly proposed symmetric parallel thinning algorithms for 3D voxel objects are very few in the literature, let us discuss each of them. Two algorithms, [31] and [32], do not preserve topology (see [27, 28]). Manzanera et al. proposed several algorithms $[4,35,36]$ that they unified in a common framework for $n$-dimensional thinning [37]. These algorithms produce curve skeletons in 2D and surface skeletons in 3D. More recently in 2008, K. Palágyi also proposed a symmetric algorithm for surface skeletons [40]. In all these works, topological and geometrical conditions cannot be separated, implying that there is no easy way to adapt these algorithms to different geometrical conditions. A symmetric algorithm, based on the framework 
of P-simple points, has been introduced in [29], we shall discuss it in the next paragraphs.

In comparison with most previous works on parallel thinning (symmetric or not), one of the most remarkable features of the proposed scheme is the separation of topological and geometrical conditions. The topological conditions are clearly stated in the framework of critical kernels and may be checked by different means (see section 5). Geometrical conditions are introduced in a generic way through a constraint set $K$ or a function $\Psi$ (see section 7 ). This brings to our scheme a flexibility that allows the user to design specific conditions, adapted to particular applications. There are only three other general strategies which allow for such a separation, namely the subfield approach, P-simple points, and $\mathcal{M}$-crucial cliques.

The subfield strategy, briefly described in the introduction, indeed allows for introducing various geometrical conditions (see $[9,33,39,38]$ ). However, the resulting skeletons are not invariant by isometries.

In the framework of P-simple points, C. Lohou and one of the authors [29] introduced a symmetric thinning scheme that they illustrated by two algorithms, one for curvilinear and one for surface skeletons. Nevertheless, it is not straightforward to use this framework for proposing more powerful thinning operators, as for example a thinning algorithm producing a minimal skeleton. This point is discussed in detail and illustrated in [12], section 5 .

Finally, the notion of $\mathcal{M}$-crucial clique $[11,12]$ indeed permits to design a $3 \mathrm{D}$ thinning scheme, which is powerful and flexible enough to produce various types of skeletons, including minimal ones. However, this scheme is less powerful than the one that we propose, which is based on $\mathcal{D}$-crucial cliques (see discussion in section 6 ). And more importantly, it has not been possible up to now to design a set of masks acting in $\mathbb{Z}^{3}$ for detecting $\mathcal{M}$-crucial cliques.

As a price to pay for symmetry, the obtained skeletons are not free of simple non-end points; in other words, they are not "thin". However, the critical kernels framework is flexible enough to permit the design of asymmetric parallel 3D thinning schemes. This is the topic of an ongoing work, where we will introduce new curvilinear and surface skeletons and compare their qualities to previously proposed ones.

\section{References}

[1] Aktouf, Z., Bertrand, G., Perroton, L.: A threedimensional holes closing algorithm. Pattern Recognition Letters 23(5), 523-531 (2002)

[2] Alexandroff, P.: Diskrete Räume. Mat. Sb 2, 501518 (1937)

[3] Alexandroff, P., Hopf, H.: Topologie, I. Springer (1935)

[4] Bernard, T., Manzanera, A.: Improved low complexity fully parallel thinning algorithm. In: Proceedings 10th International Conference on Image Analysis and Processing (ICIAP'99) (1999)

[5] Bertrand, G.: Simple points, topological numbers and geodesic neighborhoods in cubic grids. Pattern Recognition Letters 15, 1003-1011 (1994)

[6] Bertrand, G.: On P-simple points. Comptes Rendus de l'Académie des Sciences, Série Math. I(321), 1077-1084 (1995)

[7] Bertrand, G.: New notions for discrete topology. In: Discrete Geometry for Computer Imagery, LNCS, vol. 1568, pp. 218-228. Springer (1999)

[8] Bertrand, G.: On critical kernels. Comptes Rendus de l'Académie des Sciences, Série Math. I(345), 363-367 (2007)

[9] Bertrand, G., Aktouf, Z.: A three-dimensional thinning algorithm using subfields. In: Vision Geometry III, vol. 2356, pp. 113-124. SPIE (1996)

[10] Bertrand, G., Couprie, M.: A new 3D parallel thinning scheme based on critical kernels. In: Discrete Geometry for Computer Imagery, LNCS, vol. 4245, pp. 580-591. Springer (2006)

[11] Bertrand, G., Couprie, M.: Two-dimensional parallel thinning algorithms based on critical kernels. Journal of Mathematical Imaging and Vision 31(1), $35-56$ (2008)

[12] Bertrand, G., Couprie, M.: On parallel thinning algorithms: Minimal non-simple sets, $\mathrm{P}$-simple points and critical kernels. Journal of Mathematical Imaging and Vision 35(1), 23-35 (2009)

[13] Bertrand, G., Malandain, G.: A new characterization of three-dimensional simple points. Pattern Recognition Letters 15(2), 169-175 (1994) 
[14] Bing, R.: Some aspects of the topology of 3manifolds related to the Poincaré conjecture. Lectures on modern mathematics II, 93-128 (1964)

[15] Blum, H.: A transformation for extracting new descriptors of shape (1967)

[16] Couprie, M.: Note on fifteen 2D parallel thinning algorithms. Internal Report, Université de Marnela-Vallée IGM2006-01 (2005)

[17] Couprie, M., Bertrand, G.: New characterizations of simple points in 2D, 3D and 4D discrete spaces. IEEE Transactions on Pattern Analysis and Machine Intelligence 31(4), 637-648 (2009)

[18] Duda, O., Hart, P., Munson, J.: Graphical data processing research study and exterimental investigation. Tech. Rep. AD650926 (1967)

[19] Giblin, P.: Graphs, Surfaces and Homology. Chapman and Hall (1981)

[20] Golay, J.: Hexagonal parallel pattern transformations. IEEE Transactions on Computers pp. 733$740(1969)$

[21] Kong, T.: On the problem of determining whether a parallel reduction operator for n-dimensional binary images always preserves topology. In: procs. SPIE Vision Geometry II, vol. 2060, pp. 69-77 (1993)

[22] Kong, T.Y.: On topology preservation in 2-D and 3-D thinning. International Journal on Pattern Recognition and Artificial Intelligence 9, 813-844 (1995)

[23] Kong, T.Y.: Topology-preserving deletion of 1's from 2-, 3- and 4-dimensional binary images. In: Discrete Geometry for Computer Imagery, $L N C S$, vol. 1347, pp. 3-18. Springer (1997)

[24] Kong, T.Y.: Minimal non-simple and minimal noncosimple sets in binary images on cell complexes. In: Discrete Geometry for Computer Imagery, LNCS, vol. 4245, pp. 169-188. Springer (2006)

[25] Kong, T.Y., Rosenfeld, A.: Digital topology: introduction and survey. Comp. Vision, Graphics and Image Proc. 48, 357-393 (1989)

[26] Kovalevsky, V.: Finite topology as applied to image analysis. Computer Vision, Graphics and Image Processing 46, 141-161 (1989)
[27] Lohou, C.: Contribution à l'analyse topologique des images : étude d'algorithmes de squelettisation pour images $2 \mathrm{D}$ et $3 \mathrm{D}$, selon une approche topologie digitale ou topologie discrète. Ph.D. thesis, Université de Marne-la-Vallée (France) (2001)

[28] Lohou, C.: Detection of the non-topology preservation of Ma's 3D surface-thinning algorithm, by the use of P-simple points. Pattern Recognition Letters 29, 822-827 (2008)

[29] Lohou, C., Bertrand, G.: Two symmetrical thinning algorithms for 3D binary images. Pattern Recognition 40, 2301-2314 (2007)

[30] Ma, C.: On topology preservation in 3D thinning. Computer Vision, Graphics and Image Processing 59(3), 328-339 (1994)

[31] Ma, C.M.: A 3D fully parallel thinning algorithm for generating medial faces. Pattern Recognition Letters 16(1), 83-87 (1995)

[32] Ma, C.M., Sonka, M.: A 3D fully parallel thinning algorithm and its applications. Computer Vision and Image Understanding 64(3), 420-433 (1996)

[33] Ma, C.M., Wan, S.Y., Lee, J.D.: Three-dimensional topology preserving reduction on the 4-subfields. IEEE Transactions on Pattern Analysis and Machine Intelligence 24(12), 1594-1605 (2002)

[34] Malgouyres, R., Francés, A.: Deciding whether a simplicial 3-complex collapses to a 1-complex is NPcomplete. In: Discrete Geometry for Computer Imagery, LNCS, vol. 4992, pp. 177-188. Springer (2008)

[35] Manzanera, A., Bernard, T., Prêteux, F., Longuet, B.: Medial faces from a concise 3D thinning algorithm. In: Proceedings IEEE International Conference on Computer Vision (ICCV'99), pp. 337-343 (1999)

[36] Manzanera, A., Bernard, T., Prêteux, F., Longuet, B.: Ultra-fast skeleton based on an isotropic fully parallel algorithm. In: Proceedings Discrete Geometry for Computer Imagery (DGCI'99), Lecture Notes in Computer Science, vol. 1568, pp. 313-324. Springer (1999)

[37] Manzanera, A., Bernard, T., Prêteux, F., Longuet, B.: n-dimensional skeletonization: a unified mathematical framework. Journal of Electronic Imaging 11(1), 25-37 (2002) 
[38] Németh, G., Kardos, P., Palágyi, K.: Topology preserving 2-subfield 3D thinning algorithms. In: Signal Processing, Pattern Recognition and Applications (SPPRA 2010), vol. 678, pp. 311-316 (2010)

[39] Németh, G., Kardos, P., Palágyi, K.: Topology preserving 3D thinning algorithms using four and eight subfields. In: A. Campilho, M. Kamel (eds.) Image Analysis and Recognition, Lecture Notes in Computer Science, vol. 6111, pp. 316-325. Springer Berlin / Heidelberg (2010)

[40] Palágyi, K.: A 3D fully parallel surface-thinning algorithm. Theoretical Computer Science 406(1-2), 119-135 (2008)

[41] Pfaltz, J., Rosenfeld, A.: Computer representation or planar regions by their skeletons. Comm. of ACM 10, 119-125 (1967)

[42] Ronse, C.: Minimal test patterns for connectivity preservation in parallel thinning algorithms for binary digital images. Discrete Applied Mathematics 21(1), 67-79 (1988)

[43] Rosenfeld, A.: Connectivity in digital pictures. Journal of the Association for Computer Machinery 17, 146-160 (1970)

[44] Rosenfeld, A.: A characterization of parallel thinning algorithms. Information and control 29(3), 286-291 (1975)

[45] Rosenfeld, A., Pfaltz, J.: Sequential operations in digital picture processing. Journal of the Association for Computer Machinery 13, 471-494 (1966)

[46] Saha, P., Chaudhuri, B., Chanda, B., Dutta Majumder, D.: Topology preservation in 3D digital space. Pattern Recognition 27, 295-300 (1994)

[47] Whitehead, J.: Simplicial spaces, nuclei and $m$ groups. Proceedings of the London Mathematical Society 45(2), 243-327 (1939)

[48] Zeeman, E.: On the dunce hat. Topology 2, 341358 (1964)

\section{Appendix}

Proof of Th. 5.

The following result is a consequence of theorem 4.3 iii) of [8], note that this theorem holds for complexes of arbitrary dimensions.

Let $S \in \mathbb{X}^{3}$, let $R \subseteq S$, and let $T$ such that $R \subseteq T \subseteq S$. If $R^{-}$contains the critical kernel of $S$, then $T^{-}$collapses onto $R^{-}$.

Now let $X \in \mathbb{X}^{3}$ and let $Y \subseteq X$ such that $Y^{-}$contains the critical kernel of $X$.

Let $X \backslash Y=\left\{x_{1}, \ldots, x_{k}\right\}$. Thus the faces of $X \backslash Y$ are ordered according to their indices in an arbitrary way.

We set $X_{0}=X, X_{i}=X \backslash\left\{x_{1}, \ldots, x_{i}\right\}, i \in[1, k]$.

Let $i \in[1, k]$. The complex $X_{i}$ contains $Y$, thus $X_{i}^{-}$ contains all the faces which are critical for $X$. By the above result $X_{i-1}^{-}$collapses onto $X_{i}^{-}=\left[X_{i-1} \backslash\left\{x_{i}\right\}\right]^{-}$, which means that $x_{i}$ is simple for $X_{i-1}$ and that $X_{i}$ is an elementary thinning of $X_{i-1}$ (Def. 1). Thus, the xel complex $Y=X_{k}$ is a thinning of $X=X_{0}$.

\section{Proof of Th. 7 .}

Let $X \in \mathbb{X}^{3}$ and let $Y \subseteq X$.

i) Suppose $Y^{-}$contains the critical kernel of $X$. Let $Z$ such that $Y \subseteq Z \subseteq X$. Since $Z^{-}$contains the critical kernel of $X$, by Th. $5, Z$ is a thinning of $X$.

ii) Suppose $Y^{-}$does not contain the critical kernel of $X$. Then, there exists a face which is critical for $X$ in $X^{-} \backslash Y^{-}$. There exists also a face $x$ in $X^{-} \backslash Y^{-}$which is $\mathcal{M}$-critical for $X$. Then, the $\mathcal{M}$-crucial clique $C=x_{X}^{+}$is non-simple for $X$ (see [12], Th. 28, and Remark 19), i.e., the set $Z=X \backslash C$ is not a thinning of $X$. We observe that $Y \subseteq Z$. Thus, there exists $Z$ such that $Y \subseteq Z \subseteq X$, and such that $Z$ is not a thinning of $X$.

Proof of Prop. 8. We proved the proposition with the help of a computer program. All $2^{26}$ possible configurations of the neighborhood of a point $x$ in $X$ were examined, and for each of them the equivalence between definition 1 and conditions 1) and 2) was successfully tested.

Proof of Prop. 10. We proved the proposition with the help of a computer program. All $2^{16}$ possible configurations of the $\mathcal{K}$-neighborhood of a 2 -clique $C$ in $X$ were examined, and for each of them the equivalence between definition 3 and conditions 1) and 2) was successfully tested.

Proof of Prop. 11. We proved the proposition with the help of a computer program. All $2^{8}$ possible configurations of the $\mathcal{K}$-neighborhood of a 1 -clique $C$ in $X$ were examined, and for each of them the equivalence between definition 3 and the condition was successfully tested. 
Proof of Th. 16 (and Th. 15). We proved the proposition with the help of a computer program. The condition " $\mathcal{K}^{*}(C) \cap X$ is reducible" could not be checked directly because of combinatorial explosion, so we proved the property recursively with respect to the cardinality of $S=\mathcal{K}^{*}(C) \cap X$. More precisely, knowing that the proposition is trivially true for $|S|=0$, we checked it for all possible configurations of $n$ elements of $S$, for $n=1, \ldots, N$ (with $N=26,16,8$ for $d=3,2,1$ respectively, $C$ being a $d$-clique), based on the fact that the proposition was already proved for $n-1$. For simplicity, the configurations of $n$ elements out of $N$ were generated by scanning all possible $2^{N}$ configurations and selecting those with precisely $n$ elements.

Proof of Th. 17. We proved the theorem with the help of a computer program. It is trivially true when $|S|=0$ (case of a 0 -clique). We checked it for all possible configurations of $N$ elements of $S$, (with $N=26,16,8$ for $d=3,2,1$ respectively, $C$ being a $d$-clique). For each of these configurations, we tested for each simple voxel $x$ of $S$ the reducibility of $S \backslash\{x\}$, thanks to Th. 16 and to characterizations of regular cliques (Prop. 13) and simple points (Prop. 8).

\section{Proof of Th. 21.}

Let $X \in \mathbb{V}^{3}$, let $C$ be a $d$-clique which is critical for $X$, and let $x=\cap\{x \in C\}$.

Suppose $C$ is not $\mathcal{M}$-crucial for $X$. Then there exists a $d^{\prime}$-clique $D$ which is critical for $X$, and such that $x$ is a proper face of the face $y=\cap\{x \in D\}$. Thus, we have $d<d^{\prime}$ and $D$ is a proper subset of $C$.

i) Suppose $D$ is $\mathcal{D}$-crucial for $X$. It means that $C$ contains a voxel belonging to a $d^{\prime}$-clique which is $\mathcal{D}$-crucial for $X$, with $d^{\prime}>d$. Thus, $C$ is not $\mathcal{D}$-crucial for $X$.

ii) Suppose $D$ is not $\mathcal{D}$-crucial for $X$. It means that $D$ (hence also $C$ ) contains a voxel belonging to a $d^{\prime \prime}$-clique which is $\mathcal{D}$-crucial for $X$, with $d^{\prime \prime}>d^{\prime}>d$. Again, $C$ cannot be $\mathcal{D}$-crucial for $X$.

Thus, a clique which is $\mathcal{D}$-crucial for $X$ is necessarily $\mathcal{M}$-crucial for $X$. It follows that the $\mathcal{D}$-crucial kernel of $X$ is a subset of its $\mathcal{M}$-crucial kernel. 